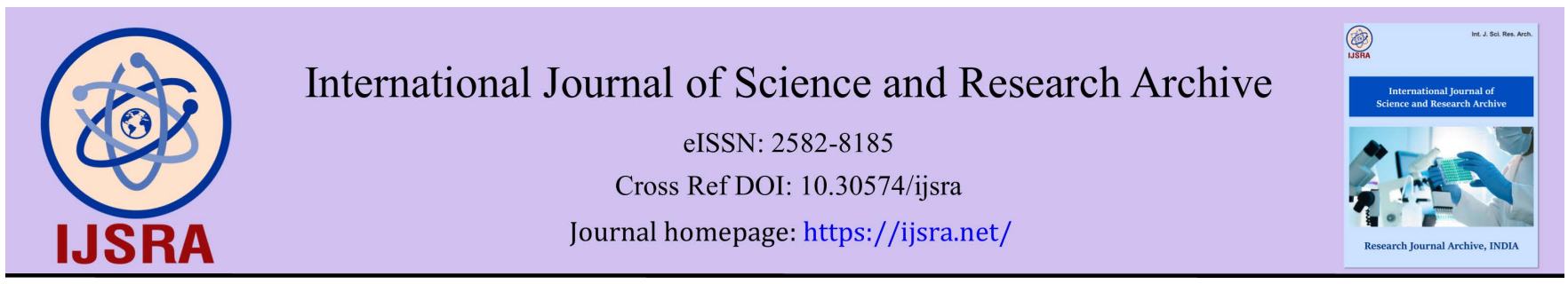

(CASE REPORT)

\title{
Treating amyotrophic lateral sclerosis with a bone marrow derived mesenchymal stem cell extracellular vesicles - A case report
}

\author{
Joshua James Crose * \\ Capitis Medical \& Aesthetics, Inc., 1917 Douglas Blvd. Ste. 89, Roseville, Ca. 95661.
}

International Journal of Science and Research Archive, 2021, 02(02), 167-171

Publication history: Received on 27 April 2021; revised on 16 May 2021; accepted on 19 May 2021

Article DOI: https://doi.org/10.30574/ijsra.2021.2.2.0058

\begin{abstract}
Amyotrophic Lateral Sclerosis (ALS) is a terminal neurodegenerative disease with only a few minimally effective treatment options. This study treats a patient with Spinal ALS with Bone Marrow Derived Mesenchymal Stem Cell Extracellular Vesicles (BM-MSCEVs) to slow or stop the progress of the disease. After 2 months and 4 treatments, the patient demonstrated improvement in ALS functional scores and most physical exam findings. BM-MSCEVs demonstrate the ability to effectively treat Spinal ALS in this study and open the door for future studies.
\end{abstract}

Keywords: Mesenchymal Stem Cells; Extracellular Vesicles; Exosomes; Amyotrophic Lateral Sclerosis; ALS; Bone Marrow

\section{Introduction}

Amyotrophic Lateral Sclerosis (ALS) is a devastating terminal disease that has perplexed medicine since its description in 1824 by Charles Bell. It was finally diagnosed and given a name in 1874 by Jean-Martin Charcot [1, 2]. ALS is the most common type of motor neuron disease and third most common neurodegenerative disease (behind Alzheimer's disease and Parkinson's disease) [2]. Approximately 4.5 per 100,000 people are living with the disease but unfortunately there has been little advancement in the understanding and treatment of it since its discovery [1,2]. The disease presents with both spinal (peripheral nerve initiated) and bulbar (central nervous system initiated) forms. Most cases of ALS (90\%-95\%) are deemed sporadic and have no known cause but both genetic and environmental factors are believed to be involved. The remaining 5-10\% of cases are classified as familial and have a genetic link to family history [3, 4, and 5]. Current diagnosis is one of exclusion based on symptoms [1-5]. Research science is now focusing on the loss of regulation and/or clearance of protein waste products, namely TDP-43, SOD1, and FUS [6, 7].

There are two FDA approved drugs for ALS. Riluzole is an oral glutamatergic neurotransmission inhibitor and decreases glutamate levels within neurons. Clinical use has shown it only slows the inevitable death of these patients by months [13-16]. Edaravone is a potent IV antioxidant and has demonstrated it slowed progression of the disease in early stages, but some conflicting studies are emerging doubting even these clinical results [2,13-16]. Unfortunately, there are no medical treatments to stop or reverse the progress of this deadly disease and access to the aforementioned treatments can be restricted and limited [1-5].

Recent Mesenchymal Stem Cell publications have focused on the potential for treating neurodegenerative pathologies [8-12]. Nisim Perets et. al. demonstrated how MSC Extracellular Vesicles (EVs) were able to concentrate in pathological areas of mice brains within 96 hours after administration [11]. H. Baharlooi et. al. hypothesized that MSCEVs will be crucial elements for delivering drugs as well as simply treating neurological pathologies due to their nature to home in on inflammation and dysfunction in the body and their ability to pass through the blood-brain barrier [12].

\footnotetext{
${ }^{*}$ Corresponding author: Joshua James Crose

Capitis Medical \& Aesthetics, Inc., 1917 Douglas Blvd. Ste. 89, Roseville, Ca. 95661.
}

Copyright (C) 2021 Author(s) retain the copyright of this article. This article is published under the terms of the Creative Commons Attribution Liscense 4.0. 
In this Case Study, A a patient with advanced ALS requiring total dependence for Activity of Daily Living (ADLs)) was selected to receive IV infusions of highly concentrated Bone Marrow-Derived Mesenchymal Stem Cell Extracellular Vesicles (BM-MSCEVs). The goal of the treatment was to slow or stop the progression of his symptoms by administering BM-MSCEVs through a peripheral IV. There already exists some precedence for the safety of IV administration of BMMSCEVs [19].

\section{Material and methods}

Dr. WS is a 54 y/o Brazilian male with spinal ALS diagnosed 2 years prior without any other co-morbidities. He initially presented with weakness in his legs and then arms four years ago that progressed to include muscle spasms and eventually loss of normal activities of daily living (ADLs). He underwent extensive testing including negative laboratory blood work, lumbar puncture, negative Brain and Spinal CT scans, and a negative MRI brain scan. Under the care of a neurologist, the patient did elicit a positive EMG and was eventually diagnosed with sporadic spinal ALS. His disease continued to progress despite his sister procuring a prescription for Riluzole from the United States. He has tried many homeopathic and alternative medicines. He was not able to procure Edaravone in Brazil, but his disease had progressed past the point of the recommended window of use based on the Clinical Trial [2]. Currently, he is wheelchair bound-and can barely lift his arms to his shoulders. He relies on his spouse and caregiver for almost all of his ADLs and is starting to slur speech and has occasional drooling with some Shortness of Breath, but he still has his mental faculties.

\subsection{Physical Exam}

- Vitals: T 36.5 Celsius, HR 83, RR 20, BP136/97, SPO2 96\% on RA.

- Gen: Cachectic appearing male wearing glasses in a wheelchair. NAD.

- HEENT: NCAT, EOMI, PERRLA, no thyromegally.

- Chest: symmetric expansion with inspiration, CTAB, no wheezing with scattered rales that cleared with a couple of breaths.

- $\quad$ CV: RRR w/o RMG, no JVD, +1/4 pulses in all extremities.

- GI: round abdomen, BS+ x 4Q, NTTP, no organomegally.

- GU: normal genitalia.

- MS: cachectic proximal and distal muscles in arms and hands with inter-digit muscle atrophy in hands. Decreased active ROM due to weakness. Lower limbs were flaccid and cachectic too.

- Skin: no rashes or overt skin lesions noted.

- Neuro: CNII-XII grossly intact with some mild slurring of his speech. He has noticeable fasciculations at rest with tremors while actively engaging his motor neurons. His abduction muscle strength is greater than his adduction strength. He cannot stand without assistance, His proximal limb strength is $3 / 5$ but he can only elevate his right arm to the level of his shoulders, his left arm is unable to lift to his shoulder. His biceps flection is $+3 / 5$ in strength.

- Lower extremities are more advanced with inability to dorsiflex his feet or toes. He has $+3 / 5$ plantar flexion strength of his feet without an elicited Babinski sign. He can extend his lower limbs against gravity, but his quadriceps muscles are $4 / 5$ strength at best.

- His sensations are grossly intact throughout these regions.

- He has 3/4 brisk patellar reflex with delayed relaxation and myoclonic oscillations (3-4) during the relaxation phase. He has $+2 / 4$ brachioradialis and $2 / 4$ triceps reflexes that are diminished on the left. Achilles reflects are $+2 / 4$ with some myoclonic oscillations (1-2).

- ALSFRS-R scores:

- Physician score: 22

- Family Member score: 21

- $\quad$ Patient score: 25

\subsection{Assessment}

Mr. WS has advanced ALS without significant respiratory involvement at this point. He is exhibiting lower and upper motor neuron involvement with slightly worse presentation on the left side. He does have preservation of sensory neuron function.

\subsection{Treatment Protocol}

Dr. WS received four separate doses of $5 \mathrm{~mL}$ of XoFlo ${ }^{\text {tm }}$ (Direct Biologics) each treatment was given intravenously every 3 weeks over a 9-week period (Time 0, week 3, week 6, week 9). His ALSFRS-R scores were reviewed from the previous 
year. Additionally, baseline scores were created by the patient, examining physician, and closest family member at time zero and at week 12 (following the last treatment). Physical Exams were performed at each visit with video recordings of the encounter documented for comparison and objective comparison.

The XoFlo ${ }^{\text {tm }}$ was diluted in NS to $10 \mathrm{~mL}$ and slowly pushed over a 10 -minute period via venous access followed by a 10 mL NS flush. The patient was observed for 15 minutes after each treatment to ensure no side effects before leaving with his family and care givers.

\section{Results}

Dr. WS responded well to the infusions with a positive gain in function. He did not exhibit any noticeable side effects from the treatment. He reported a significant decrease in muscle spasms and a significant improvement in his sleep. He did not need to take his baclofen for muscle spasms after his second treatment. Dr. WS noticed improvement in his speech and strength. He had not been able to walk with a walker for over 6 months and was able to walk with a walker for 25-50 feet regularly with minimal assistance at standing after his second treatment which persisted through his last follow up. He reported decrease pain (from muscle spasms) and was able to assist more with his ADLs.

Physical Exam changes at week 9.

\subsection{Grossly the same with exceptions}

- MS: Increased active ROM in upper extremities with ability to move fingers and hands better. Increased movement of arms and hands during speaking as well as ability to move feet and legs better.

- Neuro: CNII-XII grossly intact without slurred speech. He does not have fasciculations at rest and he has minimal tremors while actively engaging his motor neurons. His abduction muscle strength is greater than his adduction strength. He can stand with significant effort or with minimal assistance. His proximal limb strength is $+3 / 5$ and he can elevate his arms to the level of his shoulders. His biceps flection is $+3 / 5$ in strength.

- Lower extremity muscle function is improved, and he can dorsiflex his feet or toes with a $+3 / 5$ strength. He has 4/5 plantar flexion strength of his feet without an elicited Babinski sign. He can extend his lower limbs straight and his quadriceps muscles are $4 / 5$ strength.

- His sensations are grossly intact throughout these regions.

- He has 3/4 brisk patellar reflex with delayed relaxation and significant decrease in myoclonic oscillations (12 ) during the relaxation phase. He has $+2 / 4$ brachioradialis and $2 / 4$ triceps reflexes that are diminished on the left. Achilles reflects are $+2 / 4$ without myoclonic oscillations (1-2).

- $\quad$ ALSFRS-R Scores at week 12.

- Physician Score: 27

- Family Member Score: 27

- $\quad$ Patient Score: 27

\section{Discussion}

Dr. WS demonstrated significant improvement in his physical exam following his IV treatments with the BM-MSCEVs. His improvement includes almost complete resolution of tremors and muscle spasms as well as increased muscle function and strength in both upper and lower extremities. His decrease in reflex arc oscillations (myoclonus) in his patella demonstrates improvement in upper motor neuron regulation too. Subjectively he feels much better and can sleep without the use of baclofen or sleep aids and his psychological state is more upbeat. His voice is clear without slurring and he denies any swallowing difficulty. He still requires assistance with ADLs but can participate in the processes much more than before the treatment. Additionally, the ALSFRS-R scores all increased indicating gain in function. This is remarkable considering that no medicine in the world has been able to elicit this kind of response in ALS patients let alone an advanced ALS patient.

Neurodegenerative disease includes a wide range of diseases associated with progressive central nervous system damage. Stem cell-derived exosome-based therapy has been used to treat multiple sclerosis, Alzheimer's disease, Parkinson's disease, Huntington's disease, ALS and traumatic brain injury $[18,19]$.

Unlike stem cells, the paracrine factors secreted by them, including growth factors and extracellular vesicles, cross the blood-brain barrier (BBB) easily due to their small size (30-120nm) [8]. The BM-MSCEVs cross the blood-brain barrier and can locate the diseased regions and neurons in the brain and CNS. Proteomic studies have so far identified more 
than 4000 different types of proteins in EVs [20]. In addition, hundreds of different messenger RNAs and micro RNAs have been identified in BM-MSCEVs [21]. Mechanisms of action of these proteins and RNAs in treating neurodegenerative diseases include the following. The miRNA inhibits the translation of excessive and diseased proteins. The mRNA up regulates appropriate cytoplasmic shuttling to help the neurons excrete/dispose of protein toxins within the cell as well as activate proteolytic effects of internal lysosomes to help degrade built up waste products such as TDP-43. It is possible that a component of epigenetics is occurring helping up regulate or down regulate specific genes associated with ALS disease progression and activation [8-11]. Further investigations will be required to completely understand this disease altering treatment.

The BM-MSCEVs product has been used clinically thousands of times in non-ALS patients with no adverse events. A recent peer reviewed study, Exosomes Derived from Bone Marrow Mesenchymal Stem Cells as Treatment for Severe COVID-19 by Sengupta et. al., has been published on the safety and efficacy of using a bone marrow derived mesenchymal stem cell extracellular product to treat severe Covid-19 patients [17]. They found the BM-MSCEVs product was safe and highly efficacious in treating these severely ill patients. Therefore, more diverse pursuits such as this case study for ALS should be undertaken to explore other potential treatments using bone marrow derived stem cell products.

\section{Conclusion}

Bone Marrow-Derived Mesenchymal Stem Cell Extracellular Vesicles are effectively used in this case study to treat an advanced Amyotrophic Lateral Sclerosis patient. The intravenous administration of this stem cell derived product improved the ALSFRS-R scores of this patient as observed by 3 individuals over a 2 -month period without the occurrence of any negative side effects. Given the lack of treatment options for this horrible terminally progressive neurodegenerative disease and the remarkable result in this one patient, it is reasonable to pursue further evaluation of safety and efficacy of BM-MSCEVs in a larger clinical trial.

\section{Compliance with ethical standards}

\section{Acknowledgments}

I wish to thank the support of the entire family of Dr. WS who assisted with the process of coordinating the timely and appropriate treatment of the patient. I also wish to thank Dr. Sengupta who provided the costs of the product so I could make this scientific observation possible and for my wife who pushed me to restart my clinical research.

\section{Disclosure of conflict of interest}

Dr. Joshua Crose is the President of Capitis Medical \& Aesthetics, Inc. and has no Conflicts of Interest in the execution and publication of this research.

\section{Statement of ethical approval}

The investigator followed ethical procedure to perform research on human patients with internal IRB review prior to commencing any delivery of treatment.

\section{Statement of informed consent}

Informed consent was obtained from the patient before undertaking this trial.

\section{References}

[1] Mathis S, Couratier P, Julian A, Corcia P, Le Masson G. Current view and perspectives in amyotrophic lateral sclerosis. Neural Regenerative Research. 2017 Feb; 12(2): 181-184.

[2] Cruz M. Edaravone (Radicava): A Novel Agent for the Treatment of ALS, Pharmacy and Therapeutics. 2018 Jan; (1): 25-28.

[3] Hardiman O, Van Den Berg LH, Kiernan MC. Clinical diagnosis, and management of amyotrophic lateral sclerosis. Nat Rev Neurol. 2011; 7(11): 639-649.

[4] Walling AD. Amyotrophic lateral sclerosis: Lou Gehrig's disease. Am Fam Physician. 1999; 59(6): 1489-1496. 
[5] Kimura F, Fujimura C, Ishida S, Nakajima H, Furutama D, Uehara H et al. Progression rate of ALSFRS-R at time of diagnosis predicts survival time in ALS. Neurology. 2006; 66(2): 265-267.

[6] Scotter EL, Chen HJ, Shaw CE. TDP-43 Proteinopathy and ALS: Insights into Disease Mechanisms and Therapeutic Targets. Neurotherapeutics. 2015 Apr; 12(2): 352-63.

[7] Miller T, Cudkowicz M, Shaw PJ, Andersen PM, Atassi N, Bucelli RC et al. Phase 1-2 Trial of Antisense Oligonucleotide Tofersen for SOD1 ALS. The New England Journal of Medicine. 2020 July 10; 383(2):109-119.

[8] Fayazi N, Sheykhhasan M, Sleimani Asl S, Najafi R et al. Stem Cell-Derived Exosomes: A New Strategy of Neurodegenerative Disease Treatment. Molecular Neurobiology. 2021; 1-21.

[9] Guy R, Offen D. Promising Opportunities for Treating Neurodegenerative Diseases with Mesenchymal Stem CellDerived Exosomes. Biomolecules. 2020; 10(9): 13-20.

[10] Reza-Zaldivar EE, Hernandez-Sapiens MA, Minjarez B, Gutierrez-Mercado YK, Marquez-Aguirre AL, CanalesAguirre AA. Potential effects of MSC-derived exosomes in neuroplasticity in Alzheimer's disease. Frontiers in Cellular Neuroscience. 2018; 12: 317.

[11] Perets N, Betzer O, Shapira R, Brenstein S, Angel A, Sadan T et al. Golden exosomes selectively target brain pathologies in neurodegenerative and neurodevelopment disorders. Nano Letters. 2019; 19(6): 3422-3431.

[12] Baharlooi H, Azimi M, Salehi Z, Izad M. Mesenchymal Stem Cell-Derived Exosomes: A promising Therapeutic Ace Card to Address Autoimmune Diseases. International Journal of Stem Cells. 2020 Mar 30; 13(1): 13-23.

[13] Jaiswal MK. Riluzole and Edaravone: A tale of two amyotrophic lateral sclerosis drugs. Medicinal Research Reviews. 2019; 39(2): 733-748.

[14] Abe K, Aoki M, Tsuji S, Itoyama Y, Sobue G, Togo M et al. Safety and efficacy of edaravone in well-defined patients with amyotrophic lateral sclerosis: a randomized, double-blind, placebo-controlled trial. The Lancet Neurology. 2017; 16(7): 505-512.

[15] Palumbo JM, Hubble J, Apple S, Takei K, Tsuda K, Liu S et al. Post-hoc analysis of the edaravone clinical trials Study 16 and Study 19: a step toward more efficient clinical trial designs in amyotrophic lateral sclerosis. Amyotroph Lateral Scler Frontotemoral Degener. 2019 Aug; 20(5-6): 421-431.

[16] Vu M, Tortorice K, Zacher J, Dong D, Hur K, Zhang R et al. Assessment of Use and Safety of Edaravone for Amyotrophic Lateral Sclerosis in the Veterans Affairs Health Care System. JAMA 2020; 3(10): e2014645.

[17] Sengupta V, Sengupta S, Lazo A, Woods P, Nolan A, Bremer N. Exosomes Derived from Bone Marrow Mesenchymal Stem Cells as Treatment for Severe COVID-19, Stem Cells and Development Vol. 00, 2020.

[18] Gitler AD, Dhillon P, Shorter J. Neurodegenerative disease: models, mechanisms, and a new hope. Dis Model Mech. 2017; 10(5): 499-502.

[19] Magistretti PJ, Geisler FH, Schneider J, Li PA, Fiumelli H, Sipione S. Gangliosides: treatment avenues in neurodegenerative disease. Front Neurol. 2019; 10: 859.

[20] Llorente A. Mass spectrometry for the identification of protein biomarkers in urinary extracellular vesicles. In Issaq HJ, Veenstra TD, editors. Proteomic and metabolomic approaches to biomarker discovery. Proteomic and Metabolomic Approaches to Biomarker Discovery (Second Edition). Academic Press. 2020; 437-440.

[21] Munir J, Yoon J, Ryu S. Therapeutic RNA-Enriched Extracellular Vesicles: Current Approaches and Future Prospects. Cells 2020 0ct. $10^{\text {th }}$. 